\title{
Load balancing and Congestion Control in Mobile Ad-hoc Network using Ant Colony Optimization
}

\author{
Arun Kumar \\ Assistant Professor, \\ Department of Computer Engineering, \\ Maharishi Markandeshwar University, India
}

\begin{abstract}
Within few years Mobile Ad -hoc has fetched more attention due to plug and play infrastructure less network. Mobile Ad hoc wireless networks consist entirely of wireless nodes, placed together in an impromptu manner, i.e. with minimal forethought. All device/nodes have routing capabilities and forward data packets for other devices in multi hop fashion. Device/nodes can enter or leave the system at any time, so that the network topology continuously experiences revisions, modification during deployment as nodes are not fixed but moving. So routing is main problem in MANETs. Ant colony optimization is used to find the ideal solution in terms of the shortest route in a MANETs. In this paper Max-Min ant system, Multi agent Ant Colony algorithm is described. Connection is established on a multi ant net basis to balance the load in the network.

General Terms: Load Balancing, Congestion Control, Ant Colony Optimization.
\end{abstract}

\section{Keywords:}

MANET, Ant colony, optimization, routing, Ant net, Multiagent, Max-Min ant system.

\section{INTRODUCTION}

In major wireless networking environments the users devices relate either via some networking infrastructure in the form of

\author{
Vijay Kumar Katiyar, Ph.D \\ Professor and Head, \\ Department Of Computer Engineering, \\ Maharishi Markandeshwar University, India.
}

base stations and an essence of network, examples are WLANs, GSM, 3Gand 4G Networks, or directly through their intended communication partner, for e.g. using 802.11 (Fig 1) in ad-hoc mode. In comparison a mobile ad-hoc network is infrastructure less and still the devices do not need to be within one another communication range to communicate. Instead, the end users (mobile) devices also act as routers, and data packets are delivered by intermediate nodes to their final destination. MANETs are applicable in aspects where no infrastructure is available; a common example is a military scenario, rescue operations. Congestion in mobile ad-hoc networks leads to transmission delays and packet losses and causes wastage of time and energy on recovery. In the current designs, routing is not congestion adaptive. Routing may let a bottleneck happen which is detected by congestion control, but dealing with bottleneck in this reactive manner results in longer delay and unnecessary packet loss and requires significant overhead if a recent route is required. This problem becomes more evident majorly in large scale transmission of heavy traffic such as multimedia data, where bottleneck is more.

Congestion in mobile ad hoc networks leads to transmission delays and packet losses and causes wastage of time and energy on recovery. In the current designs, routing is not congestion adaptive. Routing may let a bottleneck happen which is detected by congestion control,

Figure 1: Infrastructure \& Ad-hoc Mode
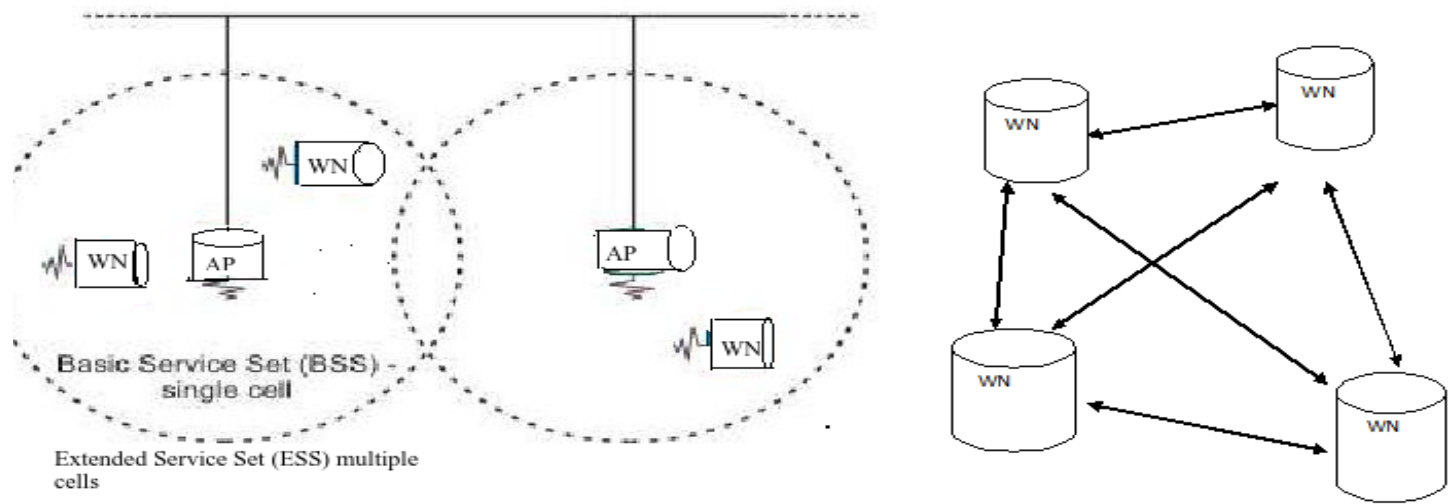
but dealing with bottleneck in this reactive manner results in longer delay and unnecessary packet loss and requires significant overhead if a recent route is required. This problem becomes more evident majorly in large scale transmission of heavy traffic such as multimedia data, where bottleneck is more plausible and the negative impact of packet loss on the service quality is of major implication. Routing should not only be cognizant of, but also be flexible to, network congestion. Routing protocols which are adaptive to the congestion status of a mobile ad - hoc network can greatly improve the network performance. Many protocols which are congestion aware and congestion adaptive have been proposed. Ant Colony optimization is technique for solving problems using graphs. Here this technique is used for congestion control in Mobile Ad-hoc Networks. The main source of motivation behind Ant Colony Optimization (ACO) and ACO routing is a behavior that is displayed by certain species of ants in nature during rummage. ACO is remarkable because each sole ant is a rather simple creature, with very limited vision and computing power, and finding the concise route among several possible routes is certainly beyond its capacity. The only way through which this difficult task can be realized is by the cooperation between the individuals in the colony. The key behind the colony level shortest route nature is the use of pheromone. It is an unstable chemical substance that is secreted by the Ants in order to influence the behavior of other Ants and of itself.

\section{LITERATURE REVIEW}

Siva Kumar.D and Bhuvaneswaran.R.S proposed Single path routing protocol, called as Ad Hoc On-demand Distance Vector that has been extensively studied for mobile ad hoc networks [1]. AODV needs a new route discovery whenever a path gaps. Such constant route discoveries originate route discovery latency. To avert such inefficiency, in the paper they present Multi agent Ants based Routing Algorithm (MARA), a new algorithm for routing in mobile ad hoc networks. Suggested hybrid protocol scales down route discovery, latency and end-to-end delay, thus providing high connectivity without requiring much of the scarce grid scope. Multi agent Ants established Routing Algorithm (MARA), is based on ideas from Ant Colony Optimization with Multi agent systems technique.

S. Prasad, Y.P.Singh, and C.S.Rai gave idea on a novel proactive algorithm to routing called Probabilistic Ant Routing, in mobile ad hoc networks, which is inspired by Ant Colony Optimization (ACO) framework and uses "ants" for route discovery, maintenance and advancement. This algorithm is supported on the mitigation of the state transition rule of ACO routing algorithm that results in maintaining higher degree of survey along with congestion awareness in the search space. This leads to reduced end-to-end delay and also lowers the overhead at high node density. Comparative experimental results for the proposed algorithm with the state of threat AODV reactive routing algorithm of the MANET are provided keeping mobility and density of nodes as the main consideration. The proposed algorithm is tested for different network sizes and node mobility. This proposed algorithm exhibits superior performance with respect to reactive AODV routing algorithm in terms of end to end delay.

\section{Hamideh Shokrani and Sam Jabbehdari presented that}

Mobile ad-hoc networks are plug play infrastructure less networks that consists of wireless, possibly mobile nodes which are organized in peer to peer fashion [3]. The highly dynamic topology, limited bandwidth availability and energy constraints make the routing problem a backbreaking one. Currently a contemporary family of algorithms emerged influence by Swarm Intelligence, which provides a unique approach to distributed optimization problems. Embryonic review have unveiled a significant deal of matching properties between the routing requirements of ad hoc networks and certain characteristics of SI, like the potential of ant colony to find a nearly optimal route amid elements. In this paper author portray a check on ant based routing algorithms for MANETs \& categorization of algorithms and outline their operation.

Al Dahoud Ali, Mohamed A. Belal and Moh'd Belal Al Zoubi represented Ant Colony Optimization (ACO) provides a Meta heuristic optimization tool and collective intelligence model to several applications such as routing and load balancing [4]. A lot of work found in the literature on using ACO in load balancing. However, apropos our mastery, there was no task divulging load balancing in distributed systems with ACO. In this study, a planned ACO algorithm for load balancing in distributed systems will be conferred. This algorithm is completely dispersed in which data is vigorously refurbished on each ant movement. Finally, the achievement of the suggested ACO algorithm is related with the work stealing approach for load balancing in distributed systems.

\section{PROTOCOLS}

Protocols we work on this paper are AODV for load balancing and for congestion avoidance in transport layer we make use of TCP Vegas.

\subsection{0n Demand Approach For Finding Routes}

\subsubsection{Ad Hoc On Demand Distance Vector (AODV)}

It uses an on demand approach for finding routes, i.e., a route is established only when it is required by a source node for transmitting data packets.

AODV routing take into consideration following walk:

- As the node enters the network it shows its existence by exchanging HELLO messages from other nodes in the network. Every mobile node regularly transmits a Hello message which all its nearest nodes receive. If node backfires to receive various Hello messages from neighbor, a connection break is detected. Hello messages are used to spot and observe links to nearest nodes.

-If Information transmission is required from origin to a specific Destination, origin node relays a Route Request (RREQ) for the terminus. Mid nodes create a route to source, when a RREQ is accepted. If the receiving node has not presented this RREQ before, it is then not the destination and does not have an up to date route to the destination, it resend the RREQ.

-Route Reply (RREP) is span if the accepted node is the end node or has a present route to the destination. RREP is unicast in a hop-by-hop method to the initial node. As the RREP propagates, each mid node fabricates a route to the destination. When the source gets the RREP, it maintains the route to the destination. 
-Initiator begins sending data through the available valid route. If multiple RREPs are found by the source, the route with the least hop count is taken as a choice. During the Transferring Route table at every node is kept by updating the clock associated with the route from source to destination.

-Provided link break is detected, a Route Error (RERR) is forward to the source of the data in a hop-by-hop fashion. As the RERR generates towards the source, each intermediate node rebut routes to any unavailable destinations. When the source of the data finds the RERR, it overrules the route and restart route discovery if necessary.

\subsubsection{Congestion Avoidance Using TCP Vegas}

TCP Vegas used as congestion avoidance algorithm it places importance on packet delay in contrast to packet loss, in signal to know rate at which packets can be send. TCP Vegas detects congestion at an initial/developing stage through increasing Round-Trip Time (RTT) values for the packets in the connection unlike other that detect congestion/bottleneck only after it has occurred through medium of packet drops.TCP Vegas algorithm depends majorly on the exact calculation of foundation/route RTT values. CASES OF RTT value:-

- Value too small throughput less than bandwidth.

- Value too large exceeds the connection.

\subsection{ACO In A Nutshell}

\subsubsection{Origin of innovation: Ants}

Ant as a sole proprietor has a very restricted potency. But as a member of a decisive colony, it becomes one robust agent, working for the growth in the colony. Every Ant lives for the colony and thrive only as a part of it. Each Ant is able to interface, grasp, cooperate, and all in all they are capable to evolve themselves and colonies on a large area. Ants manage such great affluence by enlarging the number of individuals and being unusually well planned. The self - organizing principles they are using allow a highly coordinated behavior of the colony. Ants are able to react to notable catalyst," signals that trigger a genetically conceal response. The results of these reply's can act as current important stimuli for both the insect that created them and for the different insects in the colony. This procedure is known as Stigmergy. Stigmergy is defined as the process of implied relay in a self-organizing budding system where its sole parts interface with one other by changing their neighboring domain.

\subsubsection{Optimized Trail options:}

Ants start by striding unplanned. These cannot spot the floor and have a very restricted view of what is near them. Hence, if the floor has not been traversed yet, they will just stroll and take odd resolves at every intersection. Eventually, the positions around the nest will be all surveyed. Each ant will get to realize this by the making trail done by the previous ants. Actually, they will leave after them the prominent pheromones and notify the other Ants that, the way is already searched. Concentration of pheromones relies on the strength / amount of ants which took the way, the more number of Ants taking the way, the more pheromones. The nest of a territory of Ants is attached to the food via two bridges of the identical extent. In such a setting, these begin to tour the surroundings of the nest and finally outstretch the food source. Along the trail mid food source and nest, Ants accumulate pheromones.

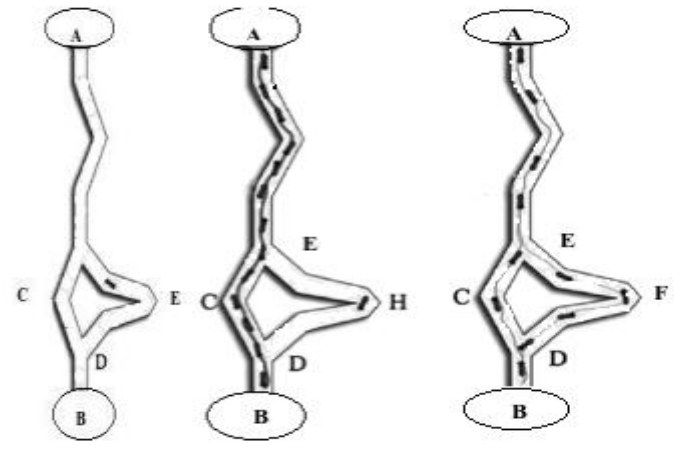

Figure 2. Source to Food Trail of Ants

Originally, every Ant irregularly chooses from one of the two bridges.

But, due to random variations, after some time one of the two bridges grants a higher concentration of pheromones than the another and, therefore, entice more Ants. It brings a further volume/quantity of pheromone on that bridge making it more attractive with the result that after some time the whole colony cross toward the use of the same bridge. Figure 2,

It gives also two paths to the food starting point, but one of them is twice longer than the other one. Here again the Ants will begin to move randomly and explore the ground. Half of ants will take shorter path than the other half according to static and probabilistic determination. The Ants engaging the shorter path will reach the food origin before the others, and leave after them the trail of pheromones. After finding the food, they will turn back and try to find the nest. At the intersection, one of the paths will accommodate pheromones in spite of the other one will be not explored. Therefore the ant that carries the food will capture, the path already surveyed, by this it means it is the way to the nest.

As the Ant is selecting the shortest way and will pursue to deposit pheromones, the trail will therefore become more attractive to others. The Ants that took the long way will have more probability to come back using the shortest way, and after some time, they will all mingle toward using it. Evidently, ants will find the shortest path through their own, without having a global/top view of the ground. By taking decision at each point according to the pheromones deposit amount, they will manage to traverse, find the food, and bring it back to the initial point, in an optimized way.

\subsubsection{Pheromones:}

It is a way through which ants communicate with each other. Vary fact that it is external and not a part of the Ants agents, confers to it an easy access for everyone. Ants create and release the hormones to communicate the memory information. The memory is preserved in without concerning the layout of the ground, the number of Ants, etc. Chemical secreted is completely independent, and still remains extremely simple.

\subsection{Ant Routing Algorithm Phases Using Ant Net:-}

- First, Route Finding Phase, in these new trails is discovered. Ant builds a path from source to destination by trail of pheromone. It makes use of ant as colony. In this we make use of two ants Forward Ant (FANT) and Back propagated Ant 
(BPANT). For new routes/paths creation we use FANT which creates trail to the source, BPANT creates trail to the destination. FANT are flooded by sender to all its neighbor nodes. Every FANT has unique sequence number to avoid copy. Node accepting FANT for first time generates record (target address, next hop, pheromone value) in its routing table. Node explain the source address of FANT as target address, address of back node as succeeding hop, and calculates pheromone value based on the count of hops the FANT used to reach the node. Node communicates FANT to neighbor, till FANT reaches destination. The destination node extracts information about load status and then destroys the FANT. BPANT is made and propagated towards source node. Through this procedure path is built and data packets sent.

- Second phase, called Route Maintenance, paths are improved during message relay. Data packets are used to keep the path, so no overhead is commenced. Pheromone variables are changing. When node Ni relays a data packet in direction of destination ND to a neighbor node $\mathrm{Nj}$, it increments the pheromone value of the entry $(\mathrm{ND}, \mathrm{Nj})$ by $\mathrm{Nd}$. The same happens in the reverse direction. The evaporation process is simulated by continuously reduction in the pheromone (catalyst) values.

- Last one handles routing failures, occurs due to node mobility mostly, and a standard issue in MANETs. Ant Routing identifies a route break through an acknowledgment not present. Links are disbanded by placing the pheromone value equal to zero. Node searches for a substitute link. Second path exists, use that. Else, neighbors are enlightened about current situation.

\subsection{Performance Metrics of Protocol:-}

In order to justify that Ant Colony Optimization Protocol is better than other routing protocols, the tools are used like Round Trip Time, Packet Delivery Proportion, Average point to point delay and Standardized Routing Load.

1. Round Trip Time (RTT): Time required by a packet to move from specified source to specified destination and vice versa. It affects latency in a network thereby reducing throughout of the network.

$\mathrm{RTT}=\mathrm{T}(\mathrm{sd})+\mathrm{T}(\mathrm{ds})$

$\mathrm{T}(\mathrm{sd})=$ Time required to move from source to destination .

$\mathrm{T}(\mathrm{ds})=$ Time required to move from destination to source.

2. Packet Delivery Proportion (PDP): Packet delivery proportion is calculated by dividing the number of packets received from the destination to the number of packets originated by source. It states the packet loss rate, which restricts the maximum output of the network. The better the delivery proportion, the more complete and accurate the routing protocol.

$\mathrm{PDP}=($ No of Packets received from Target $) /$ (No of packets originated at source).

3. Average Point-to-Point Delay: Average Point-to-Point delay is the mean time it takes a data packet to arrive at the destination in seconds. It is measured by deducting time at which first packet was transmitted by initial node from time at which first data packet arrived to destination. Embed every possible delay caused by buffering through latency, queuing at the interface progression, relay delays at MAC, propagation and transfer times. It is rhythmic in grasping the delay introduced by trail discovery.

4. Standardized Routing Load: It is the amount of routing packets relayed per data packet remitted at the destination. Each hop-wise relaying of a routing packet is counted as one transmission.

\subsection{Portray Of Protocols:-}

We describe two agents here Forward Ants (FANT) and back propagated Ants (BPANT).FANT traverse the network to find network data information and depend on probabilities to calculate the path. Back propagated Ants (BPANT) back track the steps of FANT. It makes use of data to update routing tables and other variables in the network and is discretionary. Moving agents are compact and thin packets carrying source ip address, destination ip address, Packet Id, progressive stack containing Node Id and the Node RTT. Each node contain estimated routing table. Estimated value E 1, m, d crave that node 1 takes node $\mathrm{m}$ as $\mathrm{nxt}$ node hop in order to get to destination $\mathrm{d}$.

Node draws all proximate node list by making use of single skip HELLO message packets. Msg Packets are relayed at fixed interval (continuously), each node is given expected value of $1 / \mathrm{n}$.

Where $\mathrm{n}$ is nearest nodes of node 1 . It tells we don't know anything about state of the network. Since FANT are created by source at fixed interval, FANT relay depends on avail of path to destination at node. Path to destination available means UNICAST, path to destination not available means BROADCAST.

FANT pushes Node Id and Node RTT of every closest neighbor in the stack while going towards direction. Finally when it reaches destination, information about trail/path is passed to BPANT and FANT is destroyed.

Routing Table Update:

Using ANTNET routing table are updated using quantity, r' which is obtained as:

$$
r^{\prime}=\left\{\begin{array}{l}
\frac{T}{C H} \cdot C \geq 1 . \text { if } \frac{T}{C \mu}<1 \\
1 . \quad \text { Othervise }
\end{array} \quad\right. \text {.......Equation (1) }
$$

Where $\mathrm{T}=$ trip time from the current node to the destination,

$\mu=$ average of $T$, and $C=$ scaling factor. In this we use two pheromones black and white. Individual ant lay pheromone and path is made automatically using equation 1 .

\subsubsection{MAX-MIN Ant System (MMAS):}

More performance can be attained using MAX-MIN AS in ACO. It needs three major aspects:

-To get best solution at time of algorithm run only individual ant can leave trail of pheromone. FANT may be one that get best solution under algorithm run (iteration-best ant), or that get best solution from beginning of path (global-best ant).

-For search to not become inactive range of pheromone path in every solution is restricted to an interval [ $\tau \mathrm{min} ; \tau \max$ ] 
-We intentionally initialize pheromone trails equal to $\tau \max$, in order to get broader solution.

\subsubsection{Pheromone Trail Update:}

In MMAS since individual ant is made to update pheromone trails, therefore new pheromone path update is as:

$\tau \mathrm{ij} .(\mathrm{t}+1)=. . \tau \mathrm{ij}(\mathrm{t})+\Delta \tau \mathrm{ij}$ best $\ldots \ldots .$. Equation $(2)$

Where $\Delta \tau \mathrm{ij}$ best $=1=\mathrm{f}$. (Sbest)

$=$ pheromone evaporation coefficient.

Tij = amount of pheromone deposited for a state transition ij.

It helps in finding the best trail and route finding in ACO using FANT. Data packets are further moved to sustain the given path and pheromone value becomes bigger but if value of path exceeds maximum pheromone, path is locked to control congestion using equation 2 .

\subsubsection{Multi Agent Ant Colony:}

Dearth in AODV: Route control in AODV is done by relaying HELLO messages, when acknowledgment of two HELLO messages is not made by certain node in link. Link break detected. Node that detects link break create route error message (RERR) to the origin through hop by hoop method. When RERR moves toward base node it rebuts route to any unavailable destination. When base node gets this RERR, it disapproves the route and it restart route discovery.

Ant Usage in AODV: link break control is built on fallback route discovery by ant agents. In context to protocol link fail will queue packets and find other path by using FANT and BPANT agents. These will find different path to successive node in network. It increases possibility of finding alternate trail with minimum cost. Algorithm will help in both Congestion control and load balancing. Congestion avoidance is achieved in Transport layer protocol TCP Vegas which uses RTT of FANT and BPANT to avoid bottleneck. We consider two colonies each having sequence number, here colonies are control packets. All routes to end node are familiar by base node and successive node are stocked in routing table, accessible path is check for congestion. Cost table is managed, that consist of Source ip address, Neighborhood ip address and RTT cost. Algorithm makes use of two different colonies, find two trails in order to obtain Load Balancing. Division in colonies also helps in congestion, like 1st colony ant chooses trail in which pheromone of that colony is highest, 2nd colony ant choose trail in which pheromone (catalyst) of that colony is highest thus avoiding bottleneck.

\section{ACKNOWLEDGMENT}

I thank Siva Kumar. D and Bhuvaneswaran.R.S. for laying the foundation of solving the problem of load balancing and congestion control using Ant Nets.
In short we can say that under given procedures very little focus has been put on load balancing and congestion control field. This paper discusses an routing procedure using Ant Net, Min Max Ant System and Multi agent approach based on Ant Colony Optimization. Theoretically these algorithms can help in load balancing and congestion control in the system. These algorithms will be useful in low bandwidth high latency scenario in Network and also useful if bandwidth of data send by node exceeds the provided channel.

\section{REFERENCES}

[1] Siva Kumar. D and Bhuvaneswaran.R.S. 2007. "Proposal on Multi agent Ants based Routing Algorithm for Mobile Ad-hoc Networks". IJCSNS International Journal of Computer Science and Network Security.

[2]S. Prasad, Y.P.Singh, and C.S.Rai. 2009. " Swarm Based Intelligent Routing for MANETs". IJRTE.

[3]Hamideh Shokrani, Sam Jabbehdari. 2009. "A Survey of Ant Based Routing Algorithms for Mobile Ad-hoc Networks". International Conference on Signal Processing Systems.

[4] Al Dahoud Ali, Mohamed A. Belal and Moh'd Belal Al Zoubi. 2010. "Load Balancing of Distributed Systems Based on Multiple Ant Colonies Optimization". AJASSP.

[5] C.E. Perkins and E.M. Royer. 1999. Ad-hoc on-demand distance vector routing. 2nd IEEE Workshop on Mobile Computing Systems and Applications.

[6] M.Dorigo. 2006. “Ant Colony Optimization”. University of Pretoria Etd, du Plessis J.

[7]V.Laxmi, Lavina Jain and M.S.Gaur. 2006. "Ant Colony Optimization based Routing on NS-2". International Conference on Wireless Communication and Sensor Networks (WCSN).

[8]Payman Arabshahi, Andrew Gray, Ioannis Kassabalidis and Arindam Das. 2004. "Adaptive Routing in Wireless Communication Network using Swarm Intelligence". Jet Propulsion Laboratory and University of Washington.

[9] Kwang Mong Sim and Weng Hong Sun, Member IEEE. 2003. "Ant Colony Optimization for Routing and Load Balancing: Survey and New Direction", System and Humans.

[10] Sundaram Rajagopalam and Chien-Chung Shen, 2003. "A Routing Suite for Mobile Ad hoc Networks using Swarm Intelligence". Department of Computer and Information Sciences, University of Delaware, Newark. 